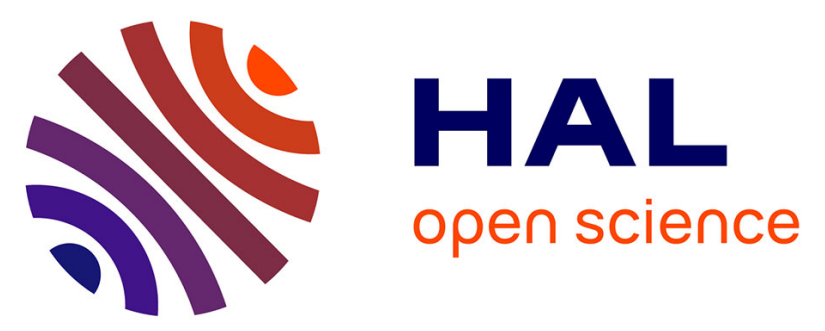

\title{
The mammalian target of rapamycin inhibitor everolimus (RAD001) in early breast cancer: results of a pre-operative study
}

E. J. Macaskill, J. M. S. Bartlett, V. S. Sabine, D. Faratian, L. Renshaw, S. White, F. M. Campbell, O. Young, L. Williams, J. S. Thomas, et al.

\section{To cite this version:}

E. J. Macaskill, J. M. S. Bartlett, V. S. Sabine, D. Faratian, L. Renshaw, et al.. The mammalian target of rapamycin inhibitor everolimus (RAD001) in early breast cancer: results of a pre-operative study. Breast Cancer Research and Treatment, 2010, 128 (3), pp.725-734. 10.1007/s10549-010-0967z . hal-00615396

\section{HAL Id: hal-00615396 https://hal.science/hal-00615396}

Submitted on 19 Aug 2011

HAL is a multi-disciplinary open access archive for the deposit and dissemination of scientific research documents, whether they are published or not. The documents may come from teaching and research institutions in France or abroad, or from public or private research centers.
L'archive ouverte pluridisciplinaire HAL, est destinée au dépôt et à la diffusion de documents scientifiques de niveau recherche, publiés ou non, émanant des établissements d'enseignement et de recherche français ou étrangers, des laboratoires publics ou privés. 
The mammalian target of rapamycin (mTOR) inhibitor everolimus (RAD001) in early breast cancer: results of a pre-operative study.

Macaskill $\mathrm{EJ}^{1,3}$, Bartlett $\mathrm{JMS}^{3}$, Sabine $\mathrm{VS}^{3}$, Faratian $\mathrm{D}^{1}$, Renshaw $\mathrm{L}^{1}$, White $\mathbf{S}^{1}$, Campbell $\mathrm{FM}^{3}$, Young $\mathrm{O}^{1}$, Williams $\mathrm{L}^{4}$, Thomas $\mathrm{JS}^{2}$, Barber $\mathrm{MD}^{1}$, Dixon $\mathrm{JM}^{1}$.

1. Breakthrough Research Unit, Western General Hospital, Edinburgh, EH4 2XU, UK.

2. Department of Pathology, Western General Hospital, Edinburgh, EH4 2XU, UK.

3. Endocrine Cancer Group, Edinburgh Cancer Research Centre, Edinburgh EH4 2XR, UK.

4. Centre for Population Health Studies, University of Edinburgh, EH8 9AG, UK.

Corresponding author:

E. Jane Macaskill

Specialist Registrar

Department of Breast Surgery

Level 6

Ninewells Hospital

Dundee DD1 9SY

UK

Tel : 01382660111 pg 5462

Email: ejanemacaskill@nhs.net

This study was funded by a research grant from Novartis Pharmaceuticals. 


\section{ABSTRACT}

Purpose: mTOR plays a key role in tumor cell cycle control, proliferation and survival. RAD001 (everolimus) is a novel macrolide that inhibits mTOR and thus downstream signaling pathways.

Experimental Design: 31 post-menopausal women with early breast cancer were given 5mg RAD001 once daily for 14 days prior to surgery. Biopsies were taken at diagnosis and at surgery (post 14 days of treatment) and assessed for immunohistochemical changes in proliferation (Ki67), apoptosis (active caspase-3), p-AKT (s473), p-S6 (s235/236 and s240/244), p-mTOR (s2448), ER, and PR.

Results: Five patients did not complete the two week treatment period due to adverse events. All adverse events were grade 1 or 2 (NCIC-CTC scale).

RAD001 treatment significantly decreased proliferation (geometric mean reduction $74 \%$ from baseline $(p=0.019))$, particularly in HER-2 positive tumors. High Ki67 pre-treatment correlated with reduction in Ki67, an increase in apoptosis, a reduction in p-Akt (cytoplasmic) and reduction in p-mTOR following treatment. Nuclear expression of p-AKT was significantly reduced with treatment. Tumors that had a reduction in Ki67 with treatment exhibited a significant reduction in cytoplasmic p-AKT. p-S6 staining was significantly reduced independently of Ki67 ( $p<0.001$ for two sites of phosphorylation).

Conclusions: RAD001 5mg/ daily is safe and tolerable in postmenopausal early breast cancer patients and inhibits the mTOR pathway and its downstream effectors, significantly reducing tumor cell proliferation. Tumors with high Ki67, high p-AKT and HER-2 positivity may be more responsive to 
mTOR inhibition with RAD001. This is the first study to report results of RAD001 5mg as a single agent in early breast cancer. 


\section{INTRODUCTION}

The mammalian target of rapamycin (mTOR) is a $289 \mathrm{kDa}$ serine/threonine protein kinase that belongs to the phosphatidylinositol-3OH kinase related kinase (PIKK) family [1-5]. mTOR is deregulated during oncogenesis resulting in disruption of a cascade of signals that regulate basic cellular functions including proliferation, apoptosis and angiogenesis [6]. Multiple mechanisms have been proposed for mTOR deregulation, including mutation of phosphatidylinositol 3-kinase (PI3K), gene amplification of AKT/PKB (protein kinase B) [7] and mutation or silencing of the phosphatase and tensin homolog (PTEN) tumor suppressor gene which, in the normal cell, acts to inhibit the activation of the PI3K-AKT cascade [8-10]. These alterations within the PI3K/PTEN axis result in hyperactivation of the AKT/ PKB family proteins, which are associated with resistance to apoptosis, increased cell growth, cell proliferation and altered cellular energy metabolism mediated via activation of mTOR [11].

mTOR exists in two distinct complexes within the mammalian cell, one consisting of mTOR, G-protein beta-subunit-like (G $\beta \mathrm{L})$ and raptor, (mTORC1) and the other includes mTOR, G $\beta$ L and rictor (mTORC2) [12-15]. These two mTOR complexes have distinct functions with mTORC1 having downstream effects including a negative feedback loop to regulate AKT through ribosomal S6 protein and insulin receptor substrate 1 (IRS1) [16]. mTORC2 (mTOR rictor complex) functions as a phosphoinositol-dependent kinase (PDK), activating AKT, and inhibition of mTORC2 reverses this effect. These distinct functions of mTORC1 and mTORC2 with respect to AKT have been established using in vitro model systems $[12-14,16]$. What remains to be 
determined is whether therapeutic inhibitors of mTOR act on mTORC1 and thus will upregulate AKT activity, or upon mTORC2 with possible inhibition of AKT.

Phospho-70S6 kinase (S6K1) and elF4E binding proteins (4E-BPs, also known as translational repressor protein PHAS-1) are two well characterized downstream effectors of mTOR activity that are activated through the mTORC1 complex [17]. Activated S6K1 phosphorylates the 40S ribosomal protein S6 to promote mRNA translation with terminal oligopyrimidine (TOP), including ribosomal proteins, elongation factors and insulin growth factor-II $[10,18,19]$

RAD001 (everolimus, Novartis) is a novel macrolide that bears a hydroxylethyl chain giving it increased polarity for oral formulation. RAD001 is licensed for use in multi-drug immunosuppression after transplantation, and has high affinity for the 12-kDa FK506-binding protein (FKBP-12); when bound to this protein RAD001 interacts with and inhibits mTOR. It is thought that the main interaction is with mTORC1 but there is evidence to suggest that with prolonged rapamycin treatment mTORC2 is also inhibited [20]. RAD001 is currently being evaluated in renal, neuroendocrine, colorectal, lung, hematological and lung tumors, as a single agent and in combination with antiangiogenic or endocrine agents following promising results in Phase I and II studies [21-24].

The aims of this clinical and biological study were to assess the safety and tolerability of RAD001 in postmenopausal women with early breast cancer, to assess the effects of 5mg RAD001 on tumor proliferation and apoptosis, and 
to investigate the effect of RAD001 as an mTOR inhibitor on the mTOR pathway. 


\section{METHODS}

Ethical approval for this study was obtained from Northern and Yorkshire MREC through the UK national application system for the clinical and biological study and use of tissue (MREC reference 04/MRE03/89). All patients gave written informed consent for all aspects of the study.

\section{Clinical Study}

The clinical study was a pre-operative 2 week study of patients with operable early breast cancer. All patients were postmenopausal females, defined as $>12$ months since last menses or in patients whose last menses was $\leq 12$ months before starting treatment, $\mathrm{FSH} \geq 35 \mathrm{IU} / \mathrm{L}$ and $\mathrm{LH} \geq 40 \mathrm{IU} / \mathrm{L}$. Patients with operable early breast cancer (T1-3, N0-1, M0) proceeding to primary surgery were eligible. Patients were not selected on the basis of tumor positivity for estrogen receptor (ER), progesterone receptor (PR) or epidermal growth factor (HER-2). Patients were dispensed 14 days of RAD001 5mg once daily so they took the last dose on the day of surgery. Patients were advised to contact the research team in the event of any side effects. A daily patient diary was completed by the patient and examined on Day 13 (preoperative admission day) to assess compliance with medication and monitoring of side effects experienced by patients, if these had not already been notified to the research team during the study period. Pre-treatment blood samples were taken on the day of enrollment in addition to the routine blood samples that had been taken within 10 days of study entry as part of preoperative assessment and screening for study suitability. 
All patients entering this study had diagnostic core biopsies taken at time of presentation with a symptomatic breast lump. The tissue was processed in accordance with local pathology department policy. Additional core biopsies were snap frozen in liquid nitrogen for expression analysis from both diagnostic core biopsies and post-treatment surgical excision specimens. The surgical excision specimen was processed by the pathology laboratory and once all diagnostic procedures completed, the tumor blocks were retrieved and sectioned for study purposes.

\section{Immunohistochemistry}

Staining was performed on all samples collected including those from patients who did not complete the full 14 days of treatment. Pre- and post-treatment sections were stained within the same staining run and anonymized prior to scoring.

Immunohistochemistry for Ki67 (MIB-1, diluted 1:50; Dako, UK), estrogen receptor (ER, NCL-L-ER-6F11/2, diluted 1:25, Novocastra Laboratories, UK) and progesterone receptor (PR, PgR636 diluted 1:100, Dako) were performed as follows: epitope retrieval was performed in citrate buffer $\mathrm{pH} 6.0$ for 6 minutes in a microwave, slides were treated with $3 \% \mathrm{H}_{2} \mathrm{O}_{2}$ for 5 minutes before washing and incubating with appropriate primary antibody at room temperature (RT) for 1 hour for ER and 30 minutes for PR and Ki67 before incubation with secondary antibody (Dako EnVision+, K5007) for 30 minutes at RT, visualized with DAB chromogen for 10 minutes at $R T$ and counterstained with haematoxylin. 
Immunohistochemistry for phospho-mTOR (serine 2448, 49F9, diluted 1:50) and phospho-S6 (serine 235/236 (clone 2211, diluted 1:400) and 240/244 (clone 2215, diluted 1:100) (all Cell Signaling Technology) were performed as follows: epitope retrieval was performed in citrate buffer $\mathrm{pH} 6.0$ at $96^{\circ} \mathrm{C}$ for 10 (p-S6) and 20 minutes (p-mTOR), slides were treated with $3 \% \mathrm{H}_{2} \mathrm{O}_{2}$ for 10 minutes (p-mTOR) and 30 minutes (p-S6), and with casein (p-mTOR) or 10\% normal goat serum ( $p$-S6) before washing and incubating over night at $4{ }^{\circ} \mathrm{C}$ with the relevant antibody before continuing with secondary antibody incubation as described above.

Immunohistochemistry for phospho-AKT (serine 473; clone 14-5 diluted 1:6, Dako, Denmark) was performed as follows: epitope retrieval was performed in TRIS/ EDTA buffer pH9.0 at $97^{\circ} \mathrm{C}$ for 20 minutes, slides were then treated with $3 \% \mathrm{H}_{2} \mathrm{O}_{2}$ for 5 minutes before washing and incubating with primary antibody for 30 minutes at RT followed by incubation with secondary antibody as before.

Immunohistochemistry for apoptosis by active caspase-3 (Abcam ab 2302, UK) was performed as described previously [25].

Immunohistochemical testing for HER-2 was performed routinely as per national guidelines. All samples scored as 2+ for HER-2 status by immunohistochemistry were tested by fluorescent in situ hybridisation (FISH). Expression of HER-2 was defined as tumors staining 3+, or 2+ and FISH positive, while all patients with tumors scored as 0 or $1+$ were considered not to express HER-2. 
All samples except Ki67 and apoptosis were scored blind using the histoscore method [26] performed by two scorers. Ki67 was counted using a previously described and validated method of scoring the percentage of stained cells as a proportion of the total cancer cells [27]. A semi-automated method of assessing apoptosis using the Ariol SL-50 image analysis system (Genetix) was employed: sections were scanned at high resolution (20x) and tumor areas manually selected. Tumor cells were scored using a previously validated algorithm. Sections were checked by a pathologist to ensure that only tumor cells were scored.

\section{Statistical analysis}

The number of patients required for this study was 30 evaluable patients to have $80 \%$ power to detect a reduction in Ki67 in $50 \%$ of patients. Analysis of variance (ANOVA) was performed to determine the significance of changes in expression of immunohistochemical results and blood results.

Results were analyzed on an intention to treat (ITT) basis, after initial ANOVA showed no difference between those completing 14 days and those who took RAD001 for less than 14 days for change in proliferation as measured by Ki67, this being the primary outcome; neither was there any difference between those completing the full course or stopping early for the other markers of the mTOR pathway assessed.

\footnotetext{
" Sabine VS, Faratian D and Bartlett JMS. Validation of cleaved caspase-3 antibody staining as a marker of apoptosis in breast cancer. Cancer Research Institute (NCRI) Cancer Conference 2008; 5 - 8 Oct 2008; Birmingham, UK. NCRI; Oct 2008. Abstract C45. Available from: www.ncri.org.uk/ncriconference/2008abstracts/C45.html
} 
ANOVA was also employed to determine differences between those that had a marked reduction in Ki67, "responders" (R), and those who did not, "nonresponders" (NR). Estimations of "responders" and "non-responders" were based upon dual observer analysis of Ki67 staining, the 95\% confidence interval for the mean difference in Ki67 counts between two observers was taken as the minimum difference which could be regarded as a change in Ki67 status between pre- and post- treatment samples [28]. Due to the nonnormal distribution of the data, Ki67 scores were log transformed prior to analysis. The change between pre and post treatment scores for each patient was calculated as the difference in the logged scores.

In order to examine the linear relationship between the immunohistochemical and blood results with the Ki67 scores, Pearson's Correlation $(\mathrm{P})$ was used. ANOVA was used to examine the change between pre and post treatment scores for all the measurements for each patient.

For the purpose of sub-group analysis data were split about the median value to differentiate between those with high and low expression for each variable. 


\section{RESULTS}

\section{Clinical study}

31 patients were recruited to this study. Five patients did not complete 14 days of treatment with RAD001 $5 \mathrm{mg}$ due to side effects. One patient was withdrawn due to a lack of study drug availability after 11 days.

The median patient age at diagnosis was 68 years (range 51-89), (Table 1) and all patients had a WHO performance scale of $0-2$. Performance status scale did not change within the treatment period for any patient.

Twelve of 31 patients reported no symptoms while taking RAD001 5mg for the two week treatment period. All adverse events reported are shown in Table 2. Five patients withdrew during the two week treatment period due to adverse events, of which two patients had more than one symptom that contributed to their withdrawal. Median duration of treatment in those that withdrew was 9.5 days (range 4-13 days). Four patients had mouth ulcers which were of sufficient impact with pain on swallowing or chewing to result in the patient withdrawing from the study, although one patient continued her medication until planned admission to hospital on Day 13. There was no correlation between tolerability to RAD001 and age or WHO performance scale.

Safety bloods were taken pre- and post-treatment with RAD001. As expected from previous data available for RAD001 treatment, there was a significant reduction in white cells, neutrophils and platelet count in the intention-to-treat population $[21,23,29,30]$. There was no link between reduction in the numbers of white cells or neutrophils with postoperative infections, nor with reduction in platelets and postoperative bleeding problems. There was a significant increase in random cholesterol with treatment with RAD001, which 
is in agreement with previous findings [21, 22, 24, 30]. There was no correlation between changes in blood parameters and reduction in Ki67 during treatment with RAD001.

\section{Biological endpoints}

All patients proceeded to surgery after the 14 day study period (Table 1). The Nottingham Prognostic Index was calculated for each patient [31]. Seven patients had an NPI in the good prognosis category, 16 were in the intermediate prognosis group and 8 were in the poor prognostic category. There was a significant correlation (Spearmans rank correlation $(r)$ ) between pre-treatment proliferation (Ki67\%) and tumor grade for both core biopsy $(r=0.59 ; 95 \% \mathrm{Cl} 0.29,0.78 ; p=0.0013)$ and excision specimens $(r=0.61 ; 95 \% \mathrm{Cl}$ $0.32,0.79 ; p=0.0009)$, see below). No correlations were found between pretreatment Ki67 and either tumor size, type, presence of LVI, node positivity or NPI.

Tumors that were of higher grade had a significantly greater reduction in proliferation after RAD001 treatment $(r=0.41 ; 95 \% \mathrm{Cl} 0.07,0.67 ; \mathrm{p}=0.024)$. Tumor size, type, presence of LVI, node positivity and NPI were not found to correlate with the change in proliferation with treatment.

Excellent intra- and inter-observer correlations were observed for histoscores (Pearson's correlation coefficient $(P)=0.984, p<0.0001$ ) and proliferation counts $(P=0.8846, p<0.0001)$.

There was a significant reduction in proliferation following treatment as assessed by Ki67 with an absolute median decrease of $8.5 \% \quad(p=0.019$; Figure 1). Using log transformed data, the post-treatment levels of Ki67 were 
$26 \%$ of those before treatment $(95 \% \mathrm{Cl} 5-56 \%)$. Those that had a high proliferation at diagnosis had the greatest absolute decrease in Ki67 (based on log transformed data $\mathrm{P}=0.36, \mathrm{p}=0.046$ ) with RAD001 treatment.

Tumor samples were split into two groups: "responders" $(R)(n=18)$ and nonresponders (NR) $(n=13)$ using the $95 \% \mathrm{Cl}$ of the absolute value for change in Ki67\% for further analysis. Pre-treatment scores for ER, PR, p-S6, p-mTOR, p-AKT and apoptosis were not significantly different for tumors that responded to RAD001 with a reduction in Ki67 counts and those that did not respond.

There was no significant change in levels of apoptosis as assessed using activated caspase-3 with RAD001 treatment for the ITT population (median pre-treatment $0.6 \%(95 \% \mathrm{Cl} 0.46,0.81)$, median post-treatment $0.22 \%(95 \% \mathrm{Cl}$ $0.15,0.68) ; p=N S$ ). There was evidence to suggest a positive linear relationship between initial Ki67 (log transformed) and the change in apoptosis $(P=0.37, p=0.04)$, in that those tumors initially high in proliferation had the greatest increase in apoptosis with RAD001 treatment (Figure 2). There was no significant difference in change in apoptosis with treatment between responders and non-responders.

While tumor ER positivity was not an entry requirement into the study, all tumors from patients entered into the study had some ER positive staining prior to treatment. There was a significant decrease in ER histoscore from pre- to post-treatment samples (mean decrease 40.5 histoscore units; $95 \% \mathrm{Cl}$ (16.9-64.2), $p=0.001$ ), with a significant difference between those with high $(n=19)$ and low ( $n=12)$ ER pre-treatment (data split about the median 240) $(p=0.009)$. Those with lower pre-treatment ER scores had a significant decrease in $\mathrm{ER}$, (mean decrease $77.5,95 \% \mathrm{Cl}$ 43.3-111.7) compared with 
those with high ER pre-treatment that had no significant change (mean decrease $17.21,95 \% \mathrm{Cl}-10.00-44.42)$. The change in ER did not correlate with pre-treatment $\mathrm{Ki} 67$ nor the change in Ki67 after treatment. There was no significant change in expression of progesterone receptor with treatment.

Eight patients had tumors that were positive for HER-2 on IHC with a score of $3+$. All those who had FISH testing for score 2+ were negative. Of those that were HER-2 positive, four were ER poor, and all were PR poor or negative. Response by Ki67 was seen in both HER-2 positive and HER-2 negative tumors. Of the 8 HER-2 positive tumors, 7 responded with a reduction in Ki67 (87.5\%), but of 23 HER-2 negative patients, only 11 had a response (47.8\%) (Figure 3). This numerical difference in response between HER-2 positive and HER-2 negative tumors did not reach statistical significance $(p=0.10)$. Tumors that were HER-2 positive had a greater reduction in p-S6 (ser 240/244 $p=0.03$ ) than HER-2 negative tumors.

For both the phosphorylation sites assessed there was a significant reduction in phosphorylation of S6 kinase with RAD001 treatment in all samples. For pS6 s235/236 the mean histoscore pre-treatment was 129.4 and this fell to 58.3 post treatment (mean decrease $71.2,95 \% \mathrm{Cl} 41.9,100.4, \mathrm{p}<0.001$ ). For p-S6 s240/244 the mean histoscore pre-treatment with RAD001 was 142.0 and 31.5 post treatment (mean decrease 110.5, 95\%Cl 82.9-138.2, $\mathrm{p}<0.001$; Figure 4). There was no evidence of a relationship between pre-treatment $p$ S6 for either site of phosphorylation or change in p-S6 with treatment and levels of proliferation either at baseline or with treatment. There was a trend toward greater reduction in p-S6 in those tumors that responded to RAD001 but this did not reach statistical significance for either s235/236 
(responders $(\mathrm{R})$ mean decrease $89.3,95 \% \mathrm{Cl}$ 51.7, 127.0; non-responders (NR) mean decrease $46.095 \% \mathrm{Cl} 1.7,90.3 ; \mathrm{p}=\mathrm{NS}$ ) or $\mathrm{s} 240 / 244$ (R mean decrease 124.5, 95\% $\mathrm{Cl} 88.5,160.6$; NR mean decrease $91.2,95 \% \mathrm{Cl} 48.8$, 133.6, $\mathrm{p}=\mathrm{NS}$ ) sites of phosphorylation. Tumors that had a greater reduction in p-S6 (ser 240/244) had a greater reduction in nuclear p-AKT after RAD001 treatment $(P=0.37, p=0.04)$.

There was no significant change in phospho-mTOR overall in the ITT population following treatment with RAD001. There was no correlation between pretreatment scores of p-mTOR and pretreatment Ki67, but there was evidence of a positive linear relationship between Ki67 pretreatment and change in $p$-mTOR with treatment $(P=0.43, p=0.0168)$, suggesting that highly proliferative tumors had an decrease in phosphorylation of mTOR after RAD001 treatment. There was no significant difference between responders and non-responders in pre-treatment or change in p-mTOR scores with RAD001.

Both nuclear and cytoplasmic staining for p-AKT was observed, and separate scores were calculated for both sites of staining. There was a significant decrease in nuclear p-AKT (mean decrease 20.9, 95\% Cl 5.02, 36.79, $\mathrm{p}=0.012)$ with RAD001 treatment. There was no evidence of a relationship between initial nuclear $p-A K T$ and change in nuclear $p$-AKT with either initial Ki67 or change in Ki67 with treatment. There was no significant difference between responders and non-responders in initial expression or change in nuclear AKT scores with RAD001 treatment.

There was no significant change in levels of cytoplasmic p-AKT with RAD001 treatment. Patients with a pre-treatment score above the median (70) fell by 
an average of $58.6(95 \% \mathrm{Cl} 25.0,92.1)$ with treatment, while the p-AKT scores of patients with pre-treatment p-AKT below median 70 had no significant change with treatment (mean increase $12.87,95 \% \mathrm{Cl}$ decrease of 21.7 to increase of $47.5, N S)$. Thus those with high pre-treatment $p$-AKT had a significant decrease in $\mathrm{p}-\mathrm{AKT}$ expression with treatment, and those with low pre-treatment expression had no change. The change between the two groups was significant $(p=0.0051)$. There was a correlation between high pretreatment levels of Ki67 and high pre-treatment cytoplasmic expression of $\mathrm{p}$ AKT $(P=0.45, p=0.0105)$, and a correlation between the change in Ki67 with treatment and change in cytoplasmic p-AKT with treatment $(P=0.45$, $\mathrm{p}=0.0106$ ), where those with a significant reduction in Ki67 also had a reduction in $\mathrm{p}-\mathrm{AKT}$ with treatment. There was a correlation between pretreatment Ki67 and the change in cytoplasmic p-AKT with treatment $(P=0.35$, $\mathrm{p}=0.0505)$, suggesting that those with high Ki67 pre-treatment had the greatest reduction in p-AKT after RAD001 treatment.

There was a significant reduction in scores for cytoplasmic p-AKT in those tumors that were classified as responding to RAD001 (mean decrease 52, $95 \% \mathrm{Cl} 19.7-84.4)$ compared with those who did not respond, in which there was no significant change (mean increase 14.8, 95\% Cl decrease 23.2increase 52.9). The difference between the groups was significant $(p=0.0105$, Figure 5).

In multivariate analysis, no pre-treatment variables were found to predict for a response to RAD001 as seen by reduction in Ki67. 


\section{DISCUSSION}

RAD001 at a dose of $5 \mathrm{mg}$ appears to be tolerable to the majority of patients. The most commonly reported side effect was mouth ulcers/ mucositis. Four of the eight patients who experienced this side effect withdrew from the study with grade I or II (mild or moderate) symptoms; these patients had pain on swallowing but were able to maintain an adequate oral intake of liquids and solids. One of these patients had mouth ulcers at the time of entry into the study and withdrew on day 9 of treatment. Four other patients experienced mouth ulcers but remained in the study as they felt that their symptoms were not interfering with their quality of life. The mouth ulcers resolved within 2 days in all patients after stopping RAD001. The rate of mouth ulcers in this study $(25 \%)$ is less than in other reported studies of RAD001 at $5 \mathrm{mg}$ or $10 \mathrm{mg}$ daily dose, which may be related to treatment duration. The selection of $5 \mathrm{mg}$ once daily as the dose for this study was on the basis of the available information at the time of recruitment beginning in 2005. Pharmacodynamic studies since published have suggested $5-10 \mathrm{mg}$ daily as an appropriate dosing schedule $[29,30]$. Reductions of S6 were the same with $5 \mathrm{mg}(\mathrm{n}=12)$ or 10mg ( $n=12)$ daily in solid advanced tumors, but there was only partial inhibition of elF-4G with $5 \mathrm{mg}$ compared to complete inhibition with $10 \mathrm{mg}$, with inter-patient variability for 4E-BP1. Side effect profiles were similar for both dose schedules, with more fatigue in the $10 \mathrm{mg}$ group. The rate of mouth ulcers was higher in this advanced study (33\%) than in our study of the same dose. In a combination study of letrozole $(2.5 \mathrm{mg})$ and RAD001 at a dose of $10 \mathrm{mg}$ daily for 16 weeks in postmenopausal women with early breast cancer more than half of patients required a dose reduction or dose interruption [23]. 
The dose of 5mg RAD001 used in our study may be more tolerable than the $10 \mathrm{mg}$ dose and remains effective as a biochemical inhibitor of the mTOR pathway.

There were significant reductions in white cells, particularly neutrophils and platelets in this study. This is of concern in the pre-operative setting, although there was no obvious increase in infective complications in the patients participating in this study, and in particular among those who had reduced neutrophil counts. Those patients with reduced neutrophil counts had the expected response to surgery with a raise in neutrophil counts observed postoperatively. Similarly, there was no increase in bleeding complications in the overall group, nor in those who had a reduction in platelets compared with the population of patients operated upon in Edinburgh Breast Unit at the time of the study.

The primary outcome measured in this pre-operative study was the change in proliferation with RAD001 treatment as measured by percentage of Ki67 positive cells. A significant decrease in Ki67 with 14 days of RAD001 at $5 \mathrm{mg}$ daily was seen in this study, confirming the effectiveness of RAD001 at reducing proliferation in breast tumors, particularly those that are highly proliferative at baseline. In a few neoadjuvant studies a reduction in Ki67 to $\leqq 1 \%$ has been used as defining "complete cell cycle response". In the current study, however, we used a significant reduction in Ki67 after treatment to define cell cycle response as the significance of reductions to $\leqq 1 \%$ remains unclear and may not reflect clinical response. We therefore felt it more appropriate to consider the absolute reductions in Ki67\% dichotomized based upon median count in line with previous studies [32, 33]. The measurement of 
Ki67 has attracted interest recently as a possible predictor of response to endocrine therapy [32]. Previous pre-operative studies performed in Edinburgh have used Ki67 as an outcome measure to allow comparison between agents and the individual efficacy of agents as anti-proliferative agents [34, 35], and this study using RAD001 has favorable results for reduction in proliferation. In the current study, tumors with the highest Ki67 pre-treatment, i.e. the most proliferative tumors, were those that had the greatest reductions in $\mathrm{Ki67}$ with treatment, the greatest absolute increase in apoptosis with treatment, and reductions in p-mTOR and p-AKT. There was also evidence of a positive correlation between change in p-AKT and Ki67 after RAD001 treatment. This would support previous findings that more aggressive or resistant cancers tend to have higher proliferation rates, and greater expression of p-AKT [36-40]. This study has found that tumors that respond to RAD001 (determined by Ki67 reduction) were those that highly expressed Ki67 and p-AKT, indicating a potential use for RAD001 in more proliferative tumors and in tumors that are resistant to other treatments, or as a combination therapy to prevent resistance or prolong sensitivity to other agents such as endocrine agents. A study of RAD001 in cell lines also found an increased sensitivity to RAD001 in cells that had high expression of $p$-AKT [41]. In the previously mentioned combination study of letrozole $2.5 \mathrm{mg}$ and RAD001 at a dose of $10 \mathrm{mg}$ daily for 16 weeks in postmenopausal women with early breast cancer [23], there was a greater reduction in Ki67 at 15 days in those receiving letrozole and RAD001 compared with those receiving letrozole alone. The extent of reduction in Ki67 correlated moderately with clinical evaluation of response. 
In a single tumor with a marked increase in proliferation (Ki67 increased from $3.6 \%-47.2 \%)$ the biomarker evidence was consistent with a lack of mTOR inhibition and upregulation of AKT signaling. Further studies would be required to explore this observation as it raises the possibility that there are tumor types that are resistant to mTOR inhibition.

There was a significant reduction of downstream effects of mTOR with RAD001 treatment demonstrated by the significant reduction in phosphorylation of $\mathrm{S} 6$ at both sites of phosphorylation. The extent of reduction at either site did not correlate with either the reduction in Ki67 or p-mTOR, although there appeared to be a correlation with reductions in nuclear $\mathrm{p}$-AKT in tumors that had a greater reduction in p-S6 with RAD001 treatment, and greater reductions in p-S6 in HER-2 positive tumors. Reductions in p-S6 have previously been shown to correlate to tumor response to mTOR inhibition in sarcoma, and may be a potential predictive marker [42].

While there was no significant change in phosphorylation of mTOR (s2448) overall, there was some evidence to suggest that those tumors that had a high Ki67 pre-treatment were more likely to have an decrease in p-mTOR after RAD001 treatment. There is debate from published data as to the mechanisms behind phosphorylation of mTOR at ser2448, but the most recent data would suggest that ser2448 is predominantly a site for mTORC1 and ser 2481 for TORC2, with p-S6 functioning as the kinase for ser2448 [43, 44].

Current understanding of the mTOR pathway and the two distinct complexes formed by mTOR with raptor (mTORC1) and rictor (mTORC2) has been based upon work with rapamycin, hence the naming of raptor (rapamycin 
associated companion to mTOR) and rictor (rapamycin insensitive companion to mTOR). One of the aims of this study was to determine the action of RAD001 upon mTOR and whether rictor is indeed insensitive to rapamycinlike mTOR inhibitors in vivo. The result that would be anticipated based on the current literature is that if only mTOR raptor is inhibited then the downregulation of mTOR, p-S6 and upregulation of p-AKT would be expected to occur by negative feedback. The negative associations of upregulation of AKT include resistance to apoptosis, increased cell growth and proliferation, increased resistance to endocrine treatment and increased risk of metastasis and reduced survival. If however, an mTOR inhibitor inhibits both mTOR raptor and mTOR rictor then it would be expected that downregulation of mTOR and p-S6, and also downregulation of p-AKT would occur. In this study it was found that in all tumors there was a significant reduction in expression of nuclear p-AKT after treatment with RAD001, indicating that both raptor and rictor complexes of mTOR are inhibited. Similarly, tumors with high cytoplasmic expression of $\mathrm{p}$-AKT pre-treatment had a reduction in $\mathrm{p}$-AKT in the cytoplasm after treatment with RAD001, and in those tumors defined as responding to RAD001 by reduction in proliferation with treatment, there was a significant reduction in expression of cytoplasmic p-AKT. However, tumors with a low p-AKT cytoplasmic expression treated with RAD001 had no change or an increase in p-AKT cytoplasmic expression with RAD001 treatment, indicating that tumors with high $\mathrm{p}$-AKT expression may respond better to RAD001 than those with low cytoplasmic p-AKT.

No association was observed between response and PI3K mutations (data not shown [45]. Although $26 \%$ of samples (8/31) harbored mutations, the 
sample size of this study is too small to draw conclusions relating to PI3K mutations and RAD001 response. In the combination study of neoadjuvant RAD001 and letrozole tumors with mutations in the PIK3CA exon 9 helical domain had a relatively small antiproliferative response to letrozole alone but a good response to RAD001 plus letrozole [23].

There was a significant reduction in ER scores with RAD001 treatment, particularly in those that had moderate to low ER levels pre-operatively. It has previously been shown that a reduction in ER can occur due to variation when comparing core biopsies and tissue sections, although no such changes have been shown for PR or Ki67 [46, 47.] This may account for the loss of ER staining particularly in those with low staining on core biopsy. There is increasing evidence for the role of $\mathrm{PI} 3 \mathrm{~K} / \mathrm{AKT}$ pathway in modulation of the ER signaling pathway [48], and this is of particular interest with the development of the concept of combining hormonal treatment with agents that block signal transduction.

Tumors were not selected for this study on the basis of tumor type, ER, PR or HER-2 status, although all tumors had some level of ER positivity. There appeared to be a greater response in tumors that were HER-2 positive, with greater reductions in $\mathrm{p}-\mathrm{S6}$. This is in keeping with results from a study of another mTOR inhibitor CCI779 (Wyeth Pharmaceuticals) in metastatic breast cancer during which no responses were seen in HER-2 positive tumors [49]. Combining a broad range of baseline tumor characteristics may have affected the results of this study, as these represent biologically different tumors with potentially different mechanisms and drivers for proliferation, and different clinical outcomes [50]. Further studies should focus on more defined 
populations to avoid this potential dilution of results, in particular in tumors with high proliferation, high p-AKT and HER-2 positivity.

The results of this study confirm that mTOR inhibition with RAD001 at $5 \mathrm{mg}$ daily dose has an effect upon ER signaling pathways as a single agent in addition to reducing proliferation. It remains to be determined whether this reduction in ER may lead to a change in responsiveness to anti-estrogens. Further studies are warranted to clarify the mechanism for this to optimize the potential for combination therapy and to clarify the role of RAD001 as a single agent. 


\section{REFERENCES}

1 Kunz J, Henriquez R, Schneider U et al (1993) Target of rapamycin in yeast, TOR2, is an essential phosphatidylinositol kinase homolog required for G1 progression. Cell 73(3): 585-596

2 Brown EJ, Albers MW, Shin TB et al (1994) A mammalian protein targeted by $\mathrm{G} 1$-arresting rapamycin-receptor complex. Nature 369: 756-758

3 Chiu MI, Katz H and Berlin V (1994) RAPT1, a mammalian homolog of yeast Tor, interacts with the FKBP12/ rapamycin complex. Proc Natl Acad Sci USA 91: 12574-12578

4 Sabatini DM, Erdjument-Bromage $\mathrm{H}$, Lui $\mathrm{M}$, Tempst $\mathrm{P}$ and Snyder $\mathrm{SH}$ (1994) RAFT1: A mammalian protein that binds to FKBP12 in a rapamycindependent fashion and is homologous to yeast TORs. Cell 78: 35-43

5 Sabers CJ, Martin MM, Brunn GJ et al (1995) Isolation of a protein target of the FKBP12-rapamycin complex in mammalian cells. J Biol Chem 270(2): 815-822

6 Chan S (2004) Targeting the mammalian target of rapamycin (mTOR): a new approach to treating cancer. Br J Cancer 91: 1420-1424

7 Vivanco I and Sawyers CL (2002) The phosphatidylinositol 3-Kinase AKT pathway in human cancer. Nat Rev Cancer 2: 489-501

8 Inoki K, Corradetti MN, and Guan KL (2005) Dysregulation of the TSCmTOR pathway in human disease. Nat Genet 37: 19-24

9 Sansal I and Sellers WR (2004) The biology and clinical relevance of the PTEN tumor suppressor pathway. J Clin Oncol 22: 2954-2963

10 Vignot S, Faivre S, Aguirre D, Raymond E (2005) mTOR-targeted therapy of cancer with rapamycin derivatives. Ann Oncol 16: 525-537

11 Hay N (2005) The Akt-mTOR tango and its relevance to cancer. Cancer Cell 8: 179-183

12 Loewith R, Jacinto E, Wullschleger S et al (2002) Two TOR complexes, only one of which is rapamycin sensitive, have distinct roles in cell growth control. Mol Cell 10: 457-468

$13 \mathrm{Kim}$ DH, Sarbassov DD, Ali SM et al (2002) mTOR interacts with raptor to form a nutrient-sensitive complex that signals to the cell growth machinery. Cell 110: 163-175

14 Sarbassov DD, Ali SM, Kim DH et al (2004) Rictor, a novel binding partner of mTOR, defines a rapamycin-insensitive and raptor-independent pathway that regulates the cytoskeleton. Curr Biol 14: 1296-1302 
15 Wullschleger S, Loewith R, Oppliger W and Hall MN (2005) Molecular organization of target of rapamycin complex 2. J Clin Biol 280(35): 3069730704

16 Sarbassov DD, Guertin DA, Ali SM and Sabatini DM (2005) Phosphorylation and regulation of Akt/ PKB by the rictor-mTOR complex. Science 307: 1098-1101

17 Burnett PE, Barrow RK, Cohen NA, Snyder SH and Sabatini DM (1998) RAFT1 phosphorylation of the translational regulators p70S6kinase and 4EBP1. Proc Natl Acad Sci USA 95: 1432-1437

18 Wullschleger S, Loewith R, Hall MN (2006) TOR signaling in growth and metabolism. Cell 124: 471-484

19 Sarbassov DD, Ali SM, Sabatini DM (2005) Growing roles for the mTOR pathway. Curr Opin Cell Biol 17: 596-603

20 Sarbassov DD, Ali SM, Sengupta S et al (2006) Prolonged Rapamycin Treatment Inhibits mTORC2 Assembly and Akt/PKB. Molecular Cell 22: 159168

21 Motzer RJ, Escudier B, Oudard S et al (2008) Efficacy of everolimus in advanced renal cell carcinoma: a double-blind, randomised, placebocontrolled phase III trial. Lancet 372: 449-456

22 Yee KWL, Zeng Z, Konopleva M et al (2006) Phase I/II study of the mammalian target of rapamycin inhibitor everolimus (RAD001) in patients with relapsed or refractory hematologic malignancies. Clin Cancer Res 12 (17): 5165-5173

23 Baselga J, Semiglazov V, van Dam P et al (2009) Phase II randomized study of neoadjuvant everolimus plus letrozole compared with placebo plus letrozole in patients with estrogen receptor-positive breast cancer. J Clin Oncol 27(16): 2630-2637

24 Kris MG, Riely GJ, Azzoli CG et al (2007) Combined inhibition of mTOR and EGFR with everolimus (RAD001) and gefitinib in patients with non-small cell lung cancer who have smoked cigarettes: A phase II trial. J Clin Oncol 25 (18s): 7575

25 Kirkegaard T, Naresh A, Sabine VS et al (2008) Expression of tumor necrosis factor alpha converting enzyme in endocrine cancers. Am J Pathol 129(5): $735-743$

26 McCarty KS, Szabo E, Flowers JL et al (1986) Use of a monoclonal antiestrogen receptor antibody in the immunohistochemical evaluation of human tumors. Cancer Res 46: 4244s-4248s 
27 Going JJ (1994) Efficiently estimated histologic cell counts. Hum Pathol 25: 333-336

28 Kirkegaard T, Edwards J, Tovey S, McGlynn LM, Krishna SN, Mukherjee R et al (2006) Observer variation in immunohistochemical analysis of protein expression, time for a change? Histopathology 48(7):787-794

29 Tabernero J, Rojo F, Calvo E et al (2008) Dose- and Schedule-dependent inhibition of the mammalian target of rapamycin pathway with everolimus: a phase I tumor pharmacodynamic study in patients with advanced solid tumors. J Clin Oncol 26: 1603-1610

30 O'Donnell A, Faivre S, Burris HA et al (2008) Phase I pharmacokinetic and pharmacodynamic study of the oral mammalian target of rapamycin inhibitor everolimus in patients with advanced solid tumors. J Clin Oncol 26: 15881595

31 Galea MH, Blamey RW, Elston CE, Ellis IO (1992) The Nottingham Prognostic Index in primary breast cancer. Breast Cancer Res Treat 22:207219

32 Dowsett M, Smith I, Ebbs SR, on behalf of the IMPACT Trialists (2005) Short-term changes in Ki-67 during neoadjuvant treatment of primary breast cancer with anastrozole or tamoxifen alone or combined correlate with recurrence-free survival. Clin Cancer Res 11: 951s-958s

33 Viale G, Giobbie-Hurder A, Regan MM et al (2008) Prognostic and Predictive Value of Centrally Reviewed Ki-67 Labeling Index in Postmenopausal Women With Endocrine-Responsive Breast Cancer: Results From Breast International Group Trial 1-98 Comparing Adjuvant Tamoxifen With Letrozole. J Clin Oncol 28: 5569-5575

34 Murray J, Young OE, Renshaw L et al (2009) A randomised study of the effects of letrozole and anastrozole on oestrogen receptor positive breast cancers in postmenopausal women. Breast Cancer Res Treat 114(3): 495501

35 Young O, Renshaw L. Macaskill EJ et al (2008) Effects of fulvestrant $750 \mathrm{mg}$ in premenopausal women with oestrogen-receptor-positive primary breast cancer. Eur J Cancer 44:391-399

36 Knuefermann C, Lu Y, Liu B et al (2003) HER2/ PI3K/ Akt activation leads to a multidrug resistance in human breast adenocarcinoma cells. Oncogene 22: $3205-3212$

37 Tokanuga E, Kataoka A, Kimura $Y$ et al (2006) The association between Akt activation and resistance to hormone therapy in metastatic breast cancer. Eur J Cancer 42: 629-635 
38 Clark AS, West K, Streicher S and Dennis PA (2002) Constitutive and inducible Akt activity promotes resistance to chemotherapy, trastuzumab, or tamoxifen in breast cancer cells. Mol Cancer Ther 1: 707-717

39 Kirkegaard T, Witton CJ, McGlynn LM et al (2005) Akt activation predicts outcome in breast cancer patients treated with tamoxifen. J Pathol 207: 139146

40 Zhou X, Tan M, Hawthorne VS et al (2004) Activation of the Akt/ Mammalian target of rapamycin/ 4E-BP1 pathway by ErbB2 overexpression predicts tumor progression in breast cancers. Clin Cancer Res 10: 6779-6788

41 Beeram M, Tan QT, Tekmal RR et al (2007) Akt-induced endocrine therapy resistance is reversed by inhibition of mTOR signaling. Ann Oncol 18(8): 1323-1328

42 Iwenofu OH, Lackman RD, Staddon AP et al (2008) Phospho-S6 ribosomal protein: a potential new predictive marker for targeted mTOR therapy. Mod Pathol 21: 231-237

43 Rosner M, Siegel N, Valli A, Fuchs C, Hengstschlager (2010) mTOR phosphorylated at s2448 binds to raptor and rictor. Amino Acids 38(1): 223228

44 Generali D, Fox SB, Brizzi MP et al (2008) Down-regulation of Phosphatidylinositol 3-Kinase/ AKT/ Molecular Target of Rapamycin metabolic pathway by primary letrozole-based therapy in human breast cancer. Clin Cancer Res 14(9): 2673-2680

45 Sabine VS, Ferguson J, Thelwell N et al (2009) Mutation of PI3KCA in post-menopausal with breast cancer and response to RAD001 treatment. Cancer Res 69 (suppl 2): 4063s

46 Burge CN, Chang HR, Apple SK (2006) Do the histologic features and results of breast cancer biomarker studies differ between core biopsy and surgical excision specimens. Breast 15: 167-172

47 lqbal S, Anderson TJ, Marson LP et al (2002) MIB-1 assessments in breast cancers. Breast 11: 252-256

48 Bhat-Nakshatri P, Wang G, Appaiah H et al (2008) AKT alters genomeside estrogen receptor alpha binding and impacts estrogen signaling in breast cancer. Mol Cell Biol 28(24): 7487-7503

49 Chan S, Scheulen ME, Johnston S et al (2005) Phase II study of Temsirolimus (CCl-779), a novel inhibitor of mTOR, in heavily pretreated patients with locally advanced or metastatic breast cancer. J Clin Oncol 23: 5314-5322 
50 Sorlie T, Perou CM, Tibshirani R, et al (2001) Gene expression patterns of breast carcinomas distinguish tumor subclasses with clinical implications. Proc Natl Acad Sci USA 98: 10869-10874

Table 1

Patient demographics

n (\%)

(unless otherwise specified)

Mean age (range, yrs)

$67.5(51-89)$

Mean tumor size (range, $\mathrm{mm}$ )

$23.9(11-41)$

Clinical tumor stage: T1

8 (25.8)

T2

$23(74.2)$

T3

0

Clinical nodal stage: NO

$28(90.3)$

N1 $3(9.7)$

N2 0

Surgery: Breast conservation

23 (74.2)

Mastectomy

Tumor type: IDC

8 (25.8)

27 (87.1)

Lobular

1 (3.2)

Mucinous

1 (3.2)

Tubular

2 (6.5)

Tumor grade: 1

8 (25.8)

$12(38.7)$

11 (35.5)

Table 1: Baseline patient and tumor demographics. All patients $(n=31)$ were postmenopausal females, with a WHO performance scale of 0-2 with early breast cancer. IDC, invasive ductal carcinoma. 
Table 2

Toxicity

Patients

Reported

Study

Mouth ulcers

Reporting

Symptom, n (\%)

8 (25.8)

6 (19.4)

3 (9.7)

2 (6.5)

2 (6.5)

2 (6.5)

2 (6.5)

1 (3.2)

1 (3.2)

Light-headed/

dizziness

Itch

1 (3.2)

Easy bruising

Joint pains
1 (3.2)

1 (3.2)
Dry mouth

Headache
Patients Withdrawing NCIC-CTC Because of Symptom, Grade n (\%)

4 (12.9)

1-2

$2(6.5)$

1

2 (6.5)

1-2

0

0

0

2

0

0

0

\section{1 \\ 1 \\ 1}

2

1

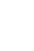

$\begin{array}{ll}0 & 1\end{array}$

0

0

Table 2: All toxicities reported in two week study period. Some patients experienced more than one symptom. 
Figure 1

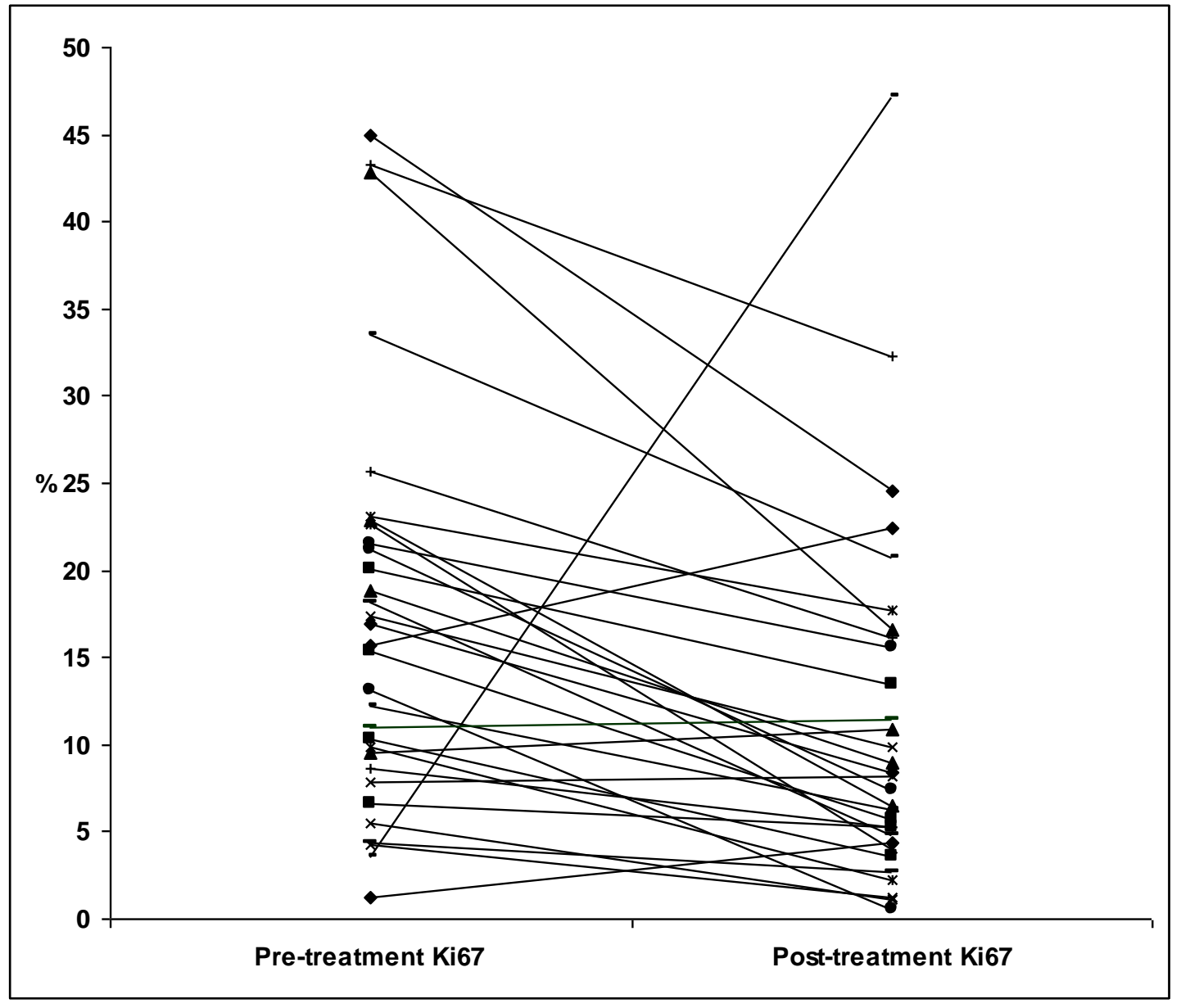

Figure 1

Individual values for Ki67 before and after treatment with RAD001 $(p=0.019)$. One tumor is noted to have increase in proliferation after treatment. 
Figure 2

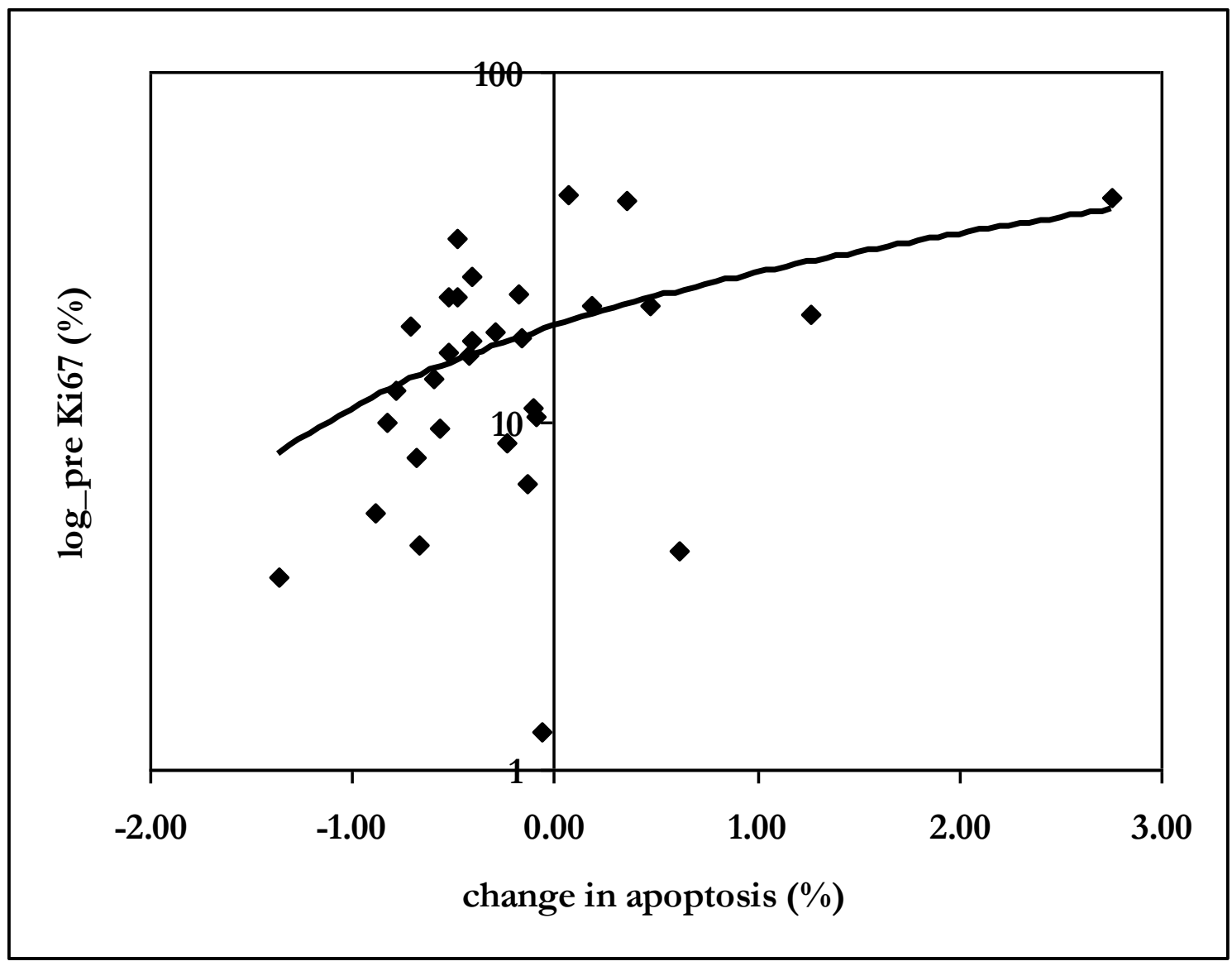

Figure 2: Correlation between pre-treatment proliferation (Ki67\%) and change in apoptosis by caspase-(\%) with RAD001 treatment suggesting that those tumors with higher pre-treatment proliferation had a greater increase in apoptosis with RAD001 treatment $(P=0.37 ; p=0.04)$. 
Figure 3

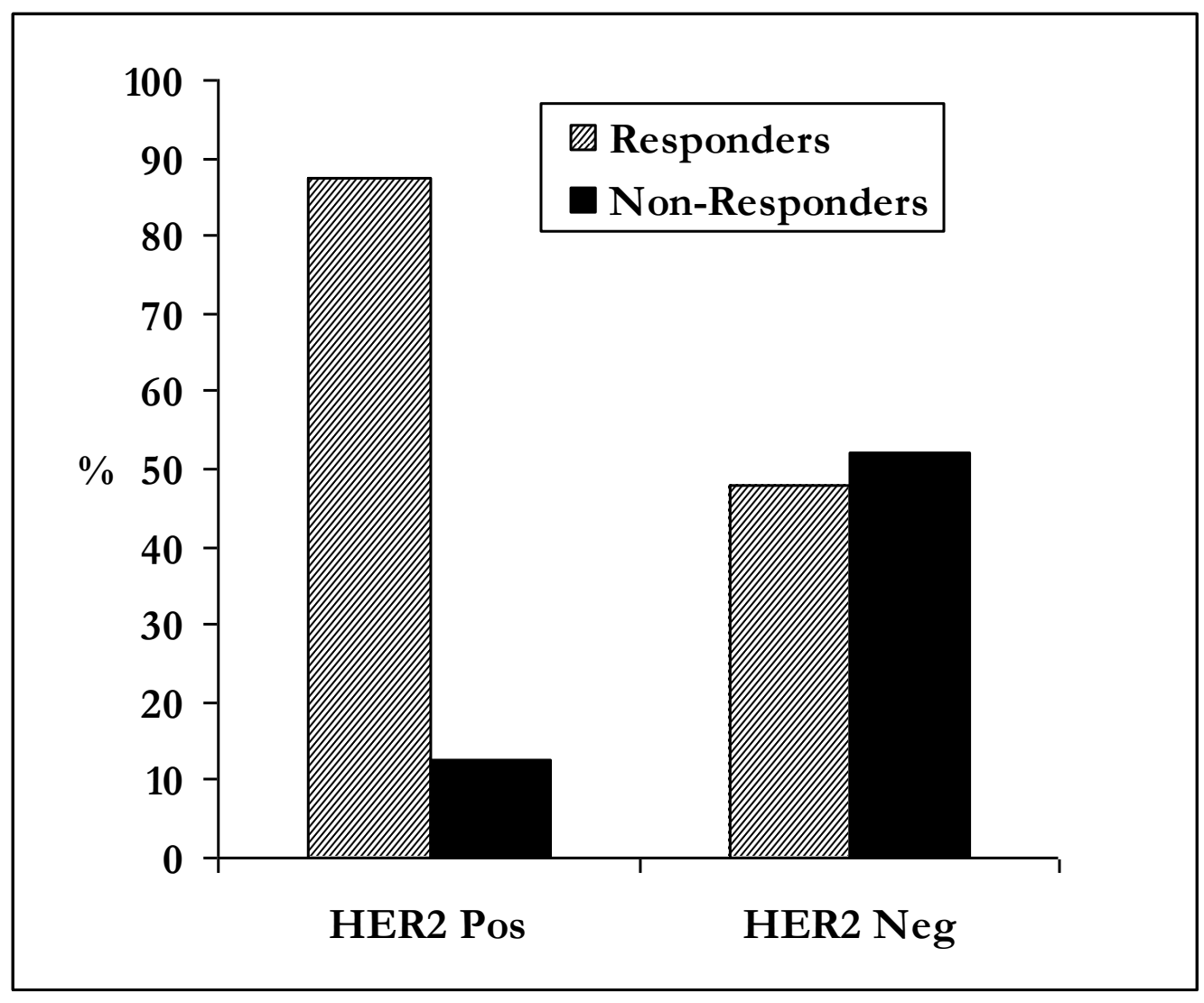

Figure 3 Response by reduction in Ki67 in HER2 positive and HER2 negative tumors showing a response in $87.5 \%(n=7)$ of HER2 positive tumors versus a response in $47.8 \%(n=11)$ of HER2 negative tumors $(p=N S)$. 
Figure 4

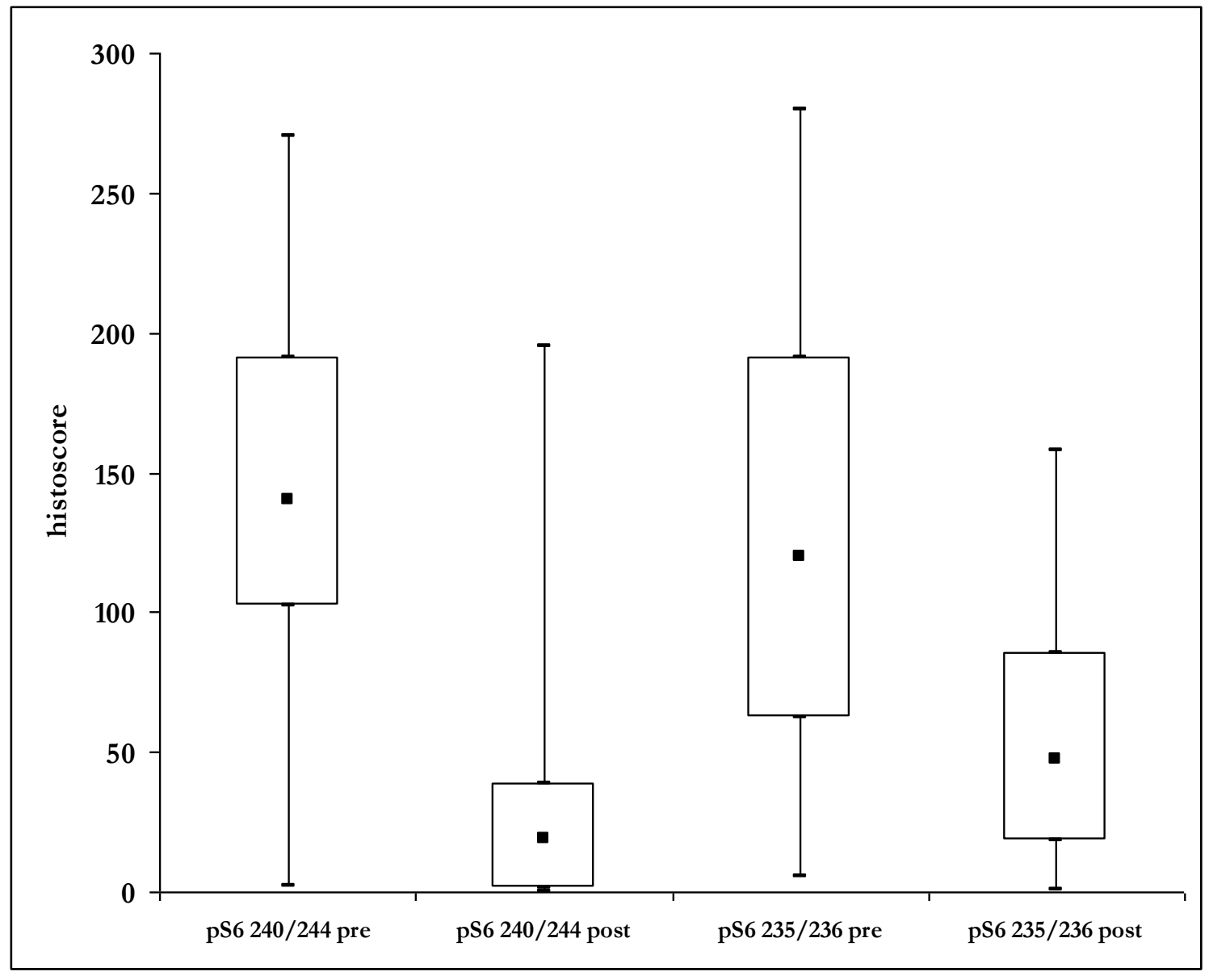

Figure 4: Change in phospho-S6 after 2 weeks treatment with RAD001 for all patients presented as box plots, demonstrating significant reduction for both sites of phosphorylation $(p<0.001)$. 
Figure 5

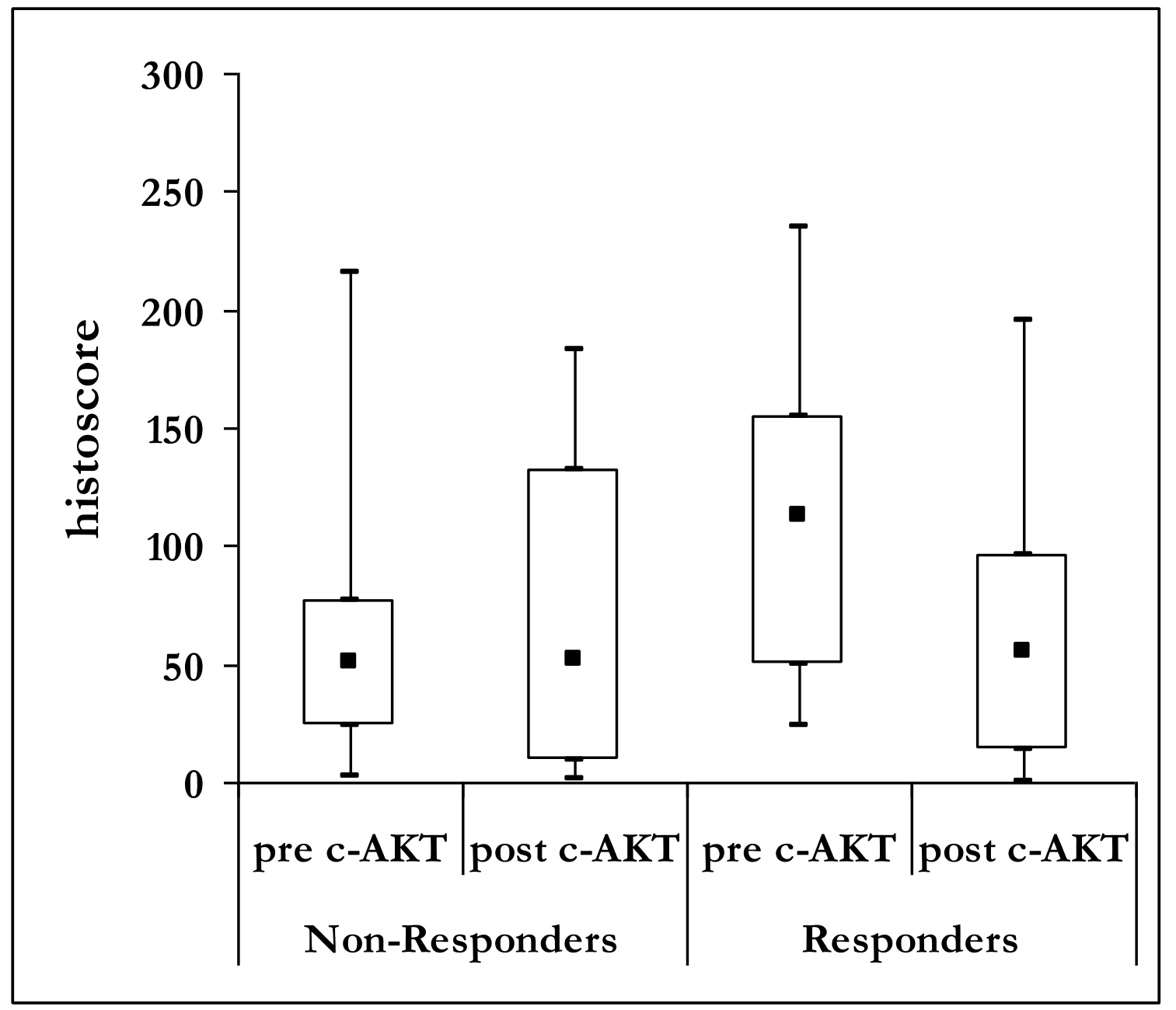

Figure 5: Change in cytoplasmic expression of pAKT after 2 weeks treatment with RAD001, divided into non-responders (left) and responders (right), demonstrating a significant difference between the two groups, with responding tumors to RAD001 having a significant reduction in expression after treatment $(p=0.0105)$, but no change in expression in those that did not respond to RAD001. 